\title{
Techniques in mechanical ventilation: principles and practice
}

\author{
J M Shneerson
}

The principles and practice of managing mechanical ventilation are often daunting to chest physicians. It is easy to see why this should be. Firstly, the physician's experience of ventilators is usually in intensive care units where the responsibility rests largely, but often to a loosely defined degree, with anaesthetists. The complex and rapidly changing medical problems of patients require frequent adjustments to the ventilator settings. In addition, there is a wide range of ventilators and the details of their performance characteristics are often unavailable to the user. The classification of ventilators is becoming more complex and more unsatisfactory as their versatility increases, and the nomenclature of the various modes of ventilation is inconsistent and confusing. ${ }^{1}$ Many of these have been inadequately assessed and their clinical indications are uncertain. Lastly, there is an increasing range of interfaces (such as nasal and face masks and new designs of tracheostomy tubes) between the ventilator and the patient to choose from and with which few doctors are familiar. ${ }^{2}$

Patients with both extrapulmonary restrictive disorders and chronic airflow obstruction are, however, being treated with ventilators in increasing numbers on medical wards and in their homes, as well as in intensive care units. Decisions about how and when to initiate ventilation, how to wean the patient from the ventilator, and how to provide long term ventilatory support are having to be faced increasingly frequently. This review addresses the principles and some of the practical issues of this type of treatment.

\section{Techniques of ventilation and their limitations}

The conventional descriptions of ventilators and ventilatory methods are complex and inconsistent. The names used by manufacturers for the method of support can be misleading and it is more important to understand what the ventilator is actually doing. Ventilators which generate a predetermined flow rate, which is usually either constant or a half sine, ramp, or reverse ramp wave form, and which have a fixed inspiratory time will deliver a predictable tidal volume. This volume preset ventilation contrasts with pressure preset ventilation in which a predetermined pressure waveform is delivered. The tidal volume will depend on the impedance to inflation and the patient's respiratory effort, but the ventilator can compensate for leaks. Some degree of leak compensation is also possible with volume preset ventilators by providing a high tidal volume which allows for the leak and setting a pressure limit during inspiration as a safety measure in case the leak decreases.

The ventilator may be time cycled from inspiration to expiration but is said to be triggered if a change in pressure or flow due to the patient's effort alters the phase of respiration. The patient's influence on ventilation can therefore range from the extreme of controlled ventilation where the tidal volume, frequency, inspiratory and expiratory times are fixed, to pressure preset ventilation with triggering of both inspiration and expiration, in which the patient can modify all of these variables. Synchronised intermittent mandatory ventilation (SIMV) combines volume preset breaths which can be triggered, with spontaneous breaths with or without a preset pressure. Other less commonly used techniques such as airway pressure release ventilation, volume or pressure controlled inverse ratio ventilation, proportional assisted ventilation and high frequency ventilation have been developed but are not in widespread use $^{3}$ and will not be considered further here.

Once the principles underlying the ventilatory methods have been established, it is important to ascertain the details of the performance characteristics of the ventilator itself. The manufacturers' manuals often give little explicit information and there is a remarkable paucity of data in the published literature about how closely different ventilators approximate to the ideal properties that would be expected from their brief technical descriptions. This void frequently leads to practical difficulties and unexpected complications in ventilator management. Ideally, the ventilator will accurately deliver the preset flow or pressure wave form in the face of varying patient mechanics and changing leaks and it should be able to provide sufficient flow for patients requiring high inspiratory flow rates. ${ }^{4}$ The inspiratory resistance of the ventilator and circuit should be low if the patient is triggering breaths and the trigger delay should be short. The expiratory resistance of the circuit should be low $^{5}$ and the extent of rebreathing within the circuit, which can vary considerably, ${ }^{6}$ should be minimal.

\section{Principles of gas exchange during ventilation}

The primary aim of mechanical ventilation is to improve alveolar gas exchange. The three components of the system - the ventilator together with its inspiratory and expiratory circuits, the patient, and the interface between the two - are in series and all influence gas and Sleep Centre, Papworth Hospital, Papworth Everard, Cambridge CB3 8RE, UK

J M Shneerson 
exchange. The minute ventilation is the product of the respiratory frequency and the tidal volume, but there are complexities even in this apparently simple relationship. Altering the frequency, for instance, changes inspiratory and expiratory times which affect the mean inspiratory flow rate in volume preset ventilators, the time for equilibration of alveolar gases and, especially in the presence of airflow obstruction, the end expiratory volume.

The tidal volume leaving the ventilator during inspiration has three destinations: (1) leaks; (2) dead space ventilation; and (3) alveolar ventilation.

\section{LEAKS}

The gas from the ventilator follows the path of lowest impedance which may be into the lungs, especially if an inspiratory effort is synchronised with the ventilator's inspiratory phase, or it may be lost through leaks in the system. These may be within the ventilator or its circuit, at the interface with or within the patient. An initial search for disconnections in the ventilator system should be made, but more commonly leaks are related to the interface with the patient. Some air usually leaks around a cuffed endotracheal or tracheostomy tube but the leak is greater with an uncuffed tube. This is usually fairly constant and can be reduced by selecting a tube which is large relative to the tracheal lumen. The leak does, however, vary according to the pressure generated in the trachea by the ventilator and the patient's efforts and the control of upper airway patency, particularly at the laryngeal level. ${ }^{78}$ Leaks around both face and nasal mask systems and, with the latter, through the mouth are often a problem. They may be associated with a raised pressure in the upper airway when this obstructs or when the patient and ventilator are uncoordinated, or with a low upper airway pressure when the muscles controlling the palate and lips fail to provide an airtight seal. This seal varies according to the degree of synchronisation between the ventilator and the patient, any underlying neuromuscular disease, the stage of sleep, and probably with the individual's intrinsic coordination of the upper airway muscles. Leaks may also occur within the patient, for instance, through a bronchopleural fistula or by air entering the oesophagus rather than the trachea. Volume preset ventilators with a high flow capacity and pressure limiting may cope adequately with leaks but, in general, they are better compensated for by pressure preset ventilators.

\section{DEAD SPACE VENTILATION}

The dead space of each ventilator circuit varies and is dependent on the length and diameter of the connecting tubing. The gas under positive pressure stretches the circuit by $2-3 \mathrm{ml} / \mathrm{cm} \mathrm{H}_{2} \mathrm{O}$ inspiratory pressure (the compressible volume). This is equivalent to an increase in dead space since this volume never reaches the patient.

The second component of the dead space is within the interface of the system with the patient. The dead space of a nasotracheal or orotracheal tube is greater than that of a tracheostomy tube and, similarly, the dead space of a face mask is greater than that of a nasal mask or mouth piece. The larger volume interfaces require more flow from the patient to trigger the ventilator because the pressure fall within them is inversely related to their volume, although this effect may be counterbalanced by the higher resistance of narrower interfaces, such as a small tracheostomy tube, which increases the effort needed to trigger the ventilator.

Within the patient, the anatomical dead space may be partially bypassed by a tracheostomy but not by non-invasive techniques such as nasal ventilation and, in general, the physiological dead space is increased rather than decreased during mechanical ventilation, particularly if the patient becomes hyperinflated.

\section{ALVEOLAR VENTILATION}

The distribution of the remaining part of the tidal volume among the alveoli is determined by factors such as the inspiratory time and the inspiratory pressure and flow pattern which result from the interaction of the ventilator and the patient's respiratory efforts, and the compliance and resistance of the alveoli. In lung diseases these are heterogeneous and an increase in the tidal volume may only overdistend the alveoli with the shortest time constants without any increase in gas exchange. Recruitment of alveoli with longer time constants may be achieved by prolonging the inspiratory time or adding an end inspiratory pause.

\section{Aims of ventilation}

Ventilation is usually only a part of the overall treatment and needs to be coordinated with the other components of the management plan. Clearly defined goals are essential whenever patients are ventilated, although it is rare for all of these to be achieved simultaneously. Compromises are inevitable and the priorities of treatment and the risks of the ventilator settings have to be carefully assessed.

The following questions should be addressed whenever ventilation is being considered.

\section{IS NORMALISATION OF THE ARTERIAL BLOOD GASES THE AIM?}

In general the answer to this question is yes, and only in exceptional circumstances should abnormal blood gas tensions be accepted. Patients with acute on chronic respiratory disorders are often managed with the aim of maintaining a degree of hypercapnia on the assumption that this is their normal state and that lowering the $\mathrm{PCO}_{2}$ will lead to apnoea and difficulties in withdrawing ventilatory assistance. This assumption should not be made unless the premorbid blood gas tensions are available and, in any case, the concentration of bicarbonate in the cerebrospinal fluid rapidly 
equilibrates with a lowered arterial $\mathrm{PCO}_{2}$ so that after 24-48 hours the sensitivity to changes in $\mathrm{PCO}_{2}$ increases. The $\mathrm{PCO}_{2}$ can usually be reduced satisfactorily by ventilation although supplemental oxygen may be required to maintain normal $\mathrm{Po}_{2} .{ }^{9}$

Occasionally, patients with severely abnormal resistance or compliance cannot be ventilated adequately, and the peak inspiratory pressure required to provide a satisfactory tidal volume may rise to potentially dangerous levels (40-50 $\mathrm{cm} \mathrm{H}_{2} \mathrm{O}$ ). The risk of barotrauma and the adverse effects on cardiac function may be sufficient to warrant a reduction in tidal volume or frequency, or both, to levels which allow the $\mathrm{PCO}_{2}$ to rise in a planned fashion (controlled hypoventilation or permissive hypercapnia)..$^{111}$ Conversely, hyperventilation is occasionally required for the specific indication of reducing intracranial pressure when a $\mathrm{PCO}_{2}$ of about $4 \mathrm{kPa}$ should be aimed for.

IS THE AIM OF VENTILATION PURELY TO REPLACE A FAILED OR INACTIVATED RESPIRATORY PUMP?

This may be the goal, for instance, in tetraplegics, during general anaesthesia with muscle relaxants in patients who have to be sedated and paralysed to tolerate ventilation or in order to avoid a raised intracranial pressure due to coughing. The patient plays a purely passive role and contributes nothing to the work of breathing. Controlled ventilation in which the patient receives a fixed tidal volume at a predetermined flow rate and with a fixed frequency is the mode of choice.

IS THE AIM OF VENTILATION TO SUPPLEMENT THE PATIENT'S OWN RESPIRATORY ACTIVITY? In this situation the ventilator is more than purely an external energy source. It is also an additional respiratory control mechanism which interacts with the patient's intrinsic respiratory control system. The respiratory cycles of both the patient and the ventilator may be partly or totally synchronised or may be uncoordinated.

Synchronisation of the patient's inspiratory efforts can trigger ventilator breaths in the assist control and SIMV modes and with pressure support. With the latter it can also augment to a variable degree the volume delivered from the ventilator, but with volume preset ventilators any continuing inspiratory activity will increase the work of breathing but will not increase the tidal volume. Triggering and inspiratory muscle activity after the onset of the ventilator's flow can occur separately or together in a single breath. ${ }^{1213}$

An uncoordinated expiratory effort during the inspiratory phase with a volume preset ventilator raises the airway pressure so that air is vented from the system at the pressure limit, thereby reducing the tidal volume. With pressure preset machines the predetermined wave form is reached with a lower tidal volume. This type of incoordination can be overcome by triggering the ventilator into expiration by air- flow rather than inspiratory time, although this type of flow triggering is only available on some pressure preset ventilators. ${ }^{1415}$

Inspiratory muscle activity during expiration slows the expiratory flow rate (braked expiration) which, while it may be beneficial in preventing airway closure, will trigger another inspiration if it leads to a negative airway pressure. If this occurs before the previous tidal volume has been fully exhaled, hyperinflation will develop (breath stacking), particularly with volume rather than pressure preset ventilators.

There do not appear to be any advantages from incoordination between the ventilator and the patient although minor degrees can occur without any distress such as in IMV, as long as gas exchange, inspiratory flow rates, respiratory frequency, and lung volume remain satisfactory. The converse of incoordination, synchrony, is usually - but not always - beneficial. Better alveolar ventilation (as well as respiratory muscle rest) may be obtained if the patient's respiration remains passive, stable, and fully supported by the ventilator.

\section{IS THE AIM OF VENTILATION TO MODIFY THE} PATIENT'S OWN RESPIRATORY ACTIVITY?

The patient's respiratory pattern can be modified considerably by the ventilator. This important but underemphasised concept conflicts with the usual advice to match the ventilator to the patient. The patient can and often must be matched to the ventilator. In its simplest form this idea is quite obvious. The respiratory pattern can be altered through relief of anxiety and discomfort once adequate ventilatory support is established. Similarly, respiration can be modified by normalising the arterial blood gases which reduces the biochemical drive to respiration. More subtle interactions are also possible. The breath to breath and intrabreath respiratory activity can be modified by, for instance, altering the inspiratory flow rate and inspiratory and expiratory time, tidal volume and lung volume. The mechanisms probably involve mechanoreceptor reflexes from the upper airway and lungs. ${ }^{16}$ The microprocessor controls on modern ventilators are becoming increasingly sophisticated, and although none can approach the flexibility of a spontaneously breathing subject, the scope for influencing the patient's respiratory activity will undoubtedly increase over the next few years.

IS THE AIM OF VENTILATION TO TREAT AS WELL AS TO SUPPORT THE PATIENT?

There is no doubt that mechanical ventilation can support the failing respiratory pump and that there is a spectrum of interaction between the patient and the machine. What is still uncertain is to what extent, and how, ventilation can improve the underlying pathophysiological changes which have led to respiratory failure. Most of the effects of ventilation discussed below are transient but, with long term nocturnal ventilation, some must persist long enough to explain the clinical and physiological 
improvements that are seen during the daytime. The most important possibilities are respiratory drive, respiratory muscle function, respiratory mechanics, and ventilation/perfusion matching.

\section{Respiratory drive}

Ventilation can influence respiratory drive by, for instance, altering the arterial $\mathrm{PCO}_{2}$ which, by lowering the concentration of bicarbonate in the cerebrospinal fluid, increases the response to hypercapnia. Relief of sleep deprivation also improves the respiratory drive through largely unknown mechanisms and, occasionally, hypoxic depression of the respiratory centres can also be reversed. The more subtle and rapidly changing interactions between the ventilator and the patient's respiratory control system which are mediated through reflex and behavioural mechanisms have been described above.

\section{Respiratory muscle function}

The concept of chronic respiratory muscle fatigue or incipient fatigue has stimulated efforts to rest the respiratory muscles and reduce the work of breathing. ${ }^{17}$ Muscle fatigue has, nevertheless, only been demonstrated in a few circumstances and it seems more likely that the central respiratory control mechanisms adapt their output to prevent it from developing. In any case rest can only be achieved if none of the ventilator breaths are triggered or augmented, which is unusual if the patient has any residual respiratory drive and muscle strength. Studies attempting to rest the respiratory muscles have, not surprisingly, shown no consistent pattern of changes. Resting the muscles to relieve or avoid fatigue should be distinguished from the use of sedation and muscle paralysis for patients whose incoordination with the ventilator cannot be controlled in any other way.

A further problem with resting the respiratory muscles is that weaning requires increasing muscle activity for which it may prove best to have exercised the muscles in order to condition or train them rather than risk disuse atrophy through rest. In practice, the aims of both respiratory muscle rest and muscle training are probably too narrow. The goal should be to optimise the pattern of respiratory muscle activity in order to enable the patient initially to be adequately ventilated by the machine and subsequently to help to regain as much respiratory muscle strength and endurance as possible in order to become independent of the ventilator. It may be best to rest the respiratory muscles during the initial stages of an acute illness or when the patient is systemically unwell, but after this a change in strategy to increase the work of breathing and improve muscle performance may assist the weaning process.

\section{Respiratory mechanics}

Most of the effects of ventilation on respiratory mechanics are transient but a few may persist after ventilation has been discontinued. A small improvement in chest wall and lung compliance may last for a few hours ${ }^{18}$ but other benefits such as reversal of abdominal paradoxical movement due to diaphragm weakness are lost after ventilation is discontinued. Alterations in upper airway resistance due to positive pressure during nasal ventilation are transient but, together with a reduction in dead space, persist with a tracheostomy as long as this is in use whether the patient is breathing spontaneously or being ventilated.

\section{Ventilation/perfusion $(\dot{V} / \dot{Q})$ matching}

Most of the effects of ventilation on V/Q matching are also transient. Regional perfusion depends on the resistance of the pulmonary arterial circulation (which is affected by gravitational forces, lung disease, vascular tone, and lung volume) and cardiac output. An increase in intrathoracic pressure reduces the intravascular blood volume, right ventricular inflow, and left ventricular outflow. These problems are therefore seen if the mean inspiratory pressure is high and if the inspiratory time is prolonged, or if the mean expiratory pressure is raised, for instance, with positive end expiratory pressure (PEEP) or continuous positive airway pressure (CPAP). Intravenous fluids may be needed to expand the intravascular volume and oxygen may be added to maintain the myocardial contractility.

It is difficult to manipulate these aspects of airway, cardiac, and vascular function to optimise $\dot{V} / \mathbf{Q}$ matching but, in general, this is usually improved by prolonging the inspiratory time with or without an end inspiratory pause ${ }^{19}$ to assist equilibration of gases within the alveoli and, if necessary, an increase in the tidal volume with or without PEEP or sighs to prevent airway closure.

\section{WHAT ARE THE OTHER ASSOCIATED AIMS OF} TREATMENT THAT ARE LINKED TO VENTILATORY SUPPORT?

Ventilatory failure is often associated with impaired bulbar function, especially in neuromuscular disorders such as motor neurone disease. The ability to cough, swallow, and speak may be reduced. In these situations, or if the level of consciousness is impaired, airway protection and access to tracheobronchial secretions with a cuffed tracheostomy or endotracheal tube may be as important as ventilatory support. In contrast, if the latter is the only necessity, a nasal or face mask or a mouth piece (or, if there is upper airway obstruction, an uncuffed tracheostomy tube) may be sufficient.

\section{IS COMPLETE WEANING THE AIM OF} VENTILATION?

This is usually the end point of ventilation, particularly if the patient has undergone surgery and does not have any chronic respiratory disorder but long term, usually nocturnal, noninvasive ventilatory support is nevertheless being increasingly provided. This is particularly 
effective in neuromuscular and skeletal disorders but is also of some benefit to carefully selected patients with chronic airflow obstruction..$^{20}$ These subjects are, in effect, only partially weaned and this long term option should be considered during the phase of continuous ventilator dependency if this is prolonged following an acute illness.

\section{Ventilation in practice}

The optimal ventilator settings for each patient are mainly determined by the metabolic requirements, respiratory drive, and mechanics. Normal subjects under general anaesthesia or after an overdose of a sedative drug, and those with little residual respiratory drive or strength - for example, tetraplegics - are easy to ventilate in the controlled mode since their mechanics are normal. Underinflation can be avoided by increasing the tidal volume of the ventilator, with or without PEEP, and hyperinflation can be prevented by reducing the tidal volume and frequency, avoiding breath stacking, lengthening the expiratory time, and adding PEEP to counteract any autoPEEP. In subjects with some residual respiratory activity, respiratory muscle rest may be achieved by reducing the respiratory drive by, for instance, increasing the inspired oxygen concentration or lowering the $\mathrm{PCO}_{2}$, increasing the level of pressure support or tidal volume, and increasing the frequency of the ventilator. Adjustment of the ventilator settings can also promote synchronisation or provoke incoordination. Synchronisation is, in general, facilitated by a low circuit inspiratory and expiratory resistance, a low trigger threshold with a short delay (which may necessitate flow cycling from expiration to inspiration and in some patients from inspiration to expiration as well), and a sufficiently fast inspiratory flow rate to meet the patient's demands. ${ }^{21}$

The ventilator can usually be easily adjusted for patients with neuromuscular and skeletal disorders despite their reduced chest wall and lung compliance. Assist control or pressure support ventilation is preferable to controlled ventilation since it allows the patient to trigger inspiration and, in the case of pressure support, to have some control over tidal volume as well. A high respiratory frequency and low tidal volume are usually required, but if this is very low either a reduction in frequency with a larger tidal volume or the addition of PEEP can overcome any tendency to basal airway closure.

Similar considerations apply to patients with diffuse pulmonary disorders such as fibrosing alveolitis, although hypercapnia is usually only a preterminal complication and ventilatory support is rarely indicated. These patients often have a greatly reduced respiratory system compliance and a strong respiratory drive, perhaps due to stimulation of pulmonary mechanoreceptors. The high respiratory drive can be at least reduced by relieving hypoxia and hypercapnia and it is important to maximise synchronisation between the patient and the ventilator. A high mean inspiratory flow rate, low tidal volume, high frequency, and appropriate adjustment of the trigger threshold may enable this to be achieved. If the patient makes expiratory efforts during inspiration the inspiratory time may need to be shortened, although this may worsen any hypoxia. Alternatively, a flow cycled ventilator with pressure support may be preferable as long as the ventilator can achieve a sufficiently high pressure to generate an adequate tidal volume.

Patients with airflow obstruction due to, for instance, chronic bronchitis or asthma pose different problems. Their expiratory flow limitation leads to an increase in end expiratory volume and pressure (autoPEEP) unless the expiratory time is sufficient for the previous tidal volume to be completely exhaled. The expiratory resistance of the ventilator and interface should be minimised by, for example, the use of a larger tracheostomy tube or a low resistance expiratory valve. In order to preserve an adequate expiratory time the inspiratory time is shortened and the respiratory frequency is reduced. Tidal volume is kept small to minimise the risk of barotrauma which is related to the peak inspiratory pressure, although some of this is dissipated along the airways and is not applied to the alveoli. PEEP may be required to overcome any autoPEEP. ${ }^{22}$

These constraints due to mechanical considerations lead to a narrow range of settings which is able to provide adequate alveolar ventilation. An important additional problem, however, is the instability of the respiratory pattern. During wakefulness this is due to behavioural influences ${ }^{23}$ such as anxiety, discomfort, and pain, but during sleep different factors operate. Firstly, there are physiological irregularities during light non-rapid eye movement sleep, usually at sleep onset, and during phasic rapid eye movement sleep. Secondly, upper airway resistance increases and diaphragmatic function is reduced, particularly during rapid eye movement sleep, so that lung volume changes and chemoreflexes and mechanoreflexes are activated. Any increase in tidal volume, however, requires a longer expiratory time to prevent an increase in end expiratory volume and autoPEEP from developing if expiratory airflow limitation is severe, whereas a reduction in tidal volume leads to a disproportionate reduction in alveolar ventilation because of the high physiological dead space in these patients. The resulting changes in blood gas tensions modify the patient's respiratory drive and may tend to perpetuate the oscillation of the respiratory pattern outside the narrow range of settings that enable adequate ventilation to be achieved.

The combination of mechanical limitations, respiratory instability, and rapidly changing blood gas tensions leads to a constantly fluctuating tidal volume, lung volume, respiratory rate, inspiratory and expiratory times and flow rates, both when the patients are clinically stable and particularly during either infective exacerbations or, in the case of asthma, acute episodes of bronchoconstriction. Synchronisation with the ventilator varies from moment to moment and it may be necessary to reduce the patient's respiratory drive by, for instance, lowering the metabolic rate (for example, by treating any infection), relieving 
hypoxia, optimising lung volume, improving air flow obstruction and considering high fat, low carbohydrate feeding to reduce carbon dioxide production. It may then be possible to synchronise the patient's respiratory effort with the ventilator which will need to be readjusted to take into account the patient's residual respiratory drive and mechanics in order to stabilise the respiratory rate and inspiratory and expiratory timing and to sustain an increased alveolar ventilation. If this cannot be achieved the alternatives are to accept a high $\mathrm{PCO}_{2}$ (controlled hypoventilation) or to sedate and paralyse the patient.

\section{Conclusion}

Mechanical ventilation of patients with respiratory failure is rarely a question of applying a machine to a passive patient. The interaction between the ventilator and the patient is complex and the properties of the ventilator and its ability to modify the patient's respiration have to be carefully considered. Clearly defined aims, an understanding of the principles and practical limitations of the equipment, and a perceptive evaluation of the patient's changing requirements are all essential if patients are to be optimally managed with ventilatory support.

1 Blanch PB, Jones M, Layton AJ, Camner N. Pressure preset ventilation. Part 1: Physiologic and mechanical preset ventilation. Part 1: Physiologic

2 Bach JR, Sortor SM, Saporito LR. Interfaces for noninvasive intermittent positive pressure ventilatory support invasive intermittent positive pressure ventilatory

3 Sassoon CSH. Positive pressure ventilation - alternate modes. Chest 1991;100:1421-9.

4 Manning HL, Molinary EJ, Leiter JC. Effect of inspiratory flow rate on respiratory sensation and pattern of breathing. Am $\mathcal{f}$ Respir Crit Care Med 1995;151:751-7.

5 Dennison FH, Taft AA, Mishoe SC, Hooker LL, Eatherly SB, Beckham RW. Analysis of resistance to gas flow in nine adult ventilator circuits. Chest 1989;96:1374-9.
6 Ferguson GT, Gilmartin M. Carbon dioxide rebreathing during $\mathrm{BiPAP}^{\mathrm{R}}$ ventilatory assistance. Am $\mathcal{f}$ Respir Crit Care Med 1995;151:1126-35.

7 Jounieaux V, Aubert G, Dury M, Delguste P, Rodenstein DO. Effects of nasal positive-pressure hyperventilation on the glottis in normal awake subjects. $\mathcal{f}$ Appl Physiol 1995; 79:176-85.

8 Jounieaux V, Aubert G, Dury M, Delguste P, Rodenstein DO. Effects of nasal positive-pressure hyperventilation on the glottis in normal sleeping subjects. $\mathcal{F}$ Appl Physiol 1995; 79:186-93.

9 Smith IE, Shneerson JM. A progressive care programme for prolonged ventilatory failure: analysis of outcome. $B r f$ prolonged ventilatory fail

10 Tuxen DV. Permissive hypercapnic ventilation. Am F Respir Crit Care Med 1994;150:870-4.

11 Feihl F, Perret C. Permissive hypercapnia - how permissive should we be? Am $\mathcal{F}$ Respir Crit Care Med 1994;150: 1722-37.

12 Sassoon CSH, Mahutte CK, Te TT, Simmons DH, Light $\mathrm{RW}$. Work of breathing and airway occlusion pressure during assist-mode mechanical ventilation. Chest 1988;93: $571-6$.

13 Flick GR, Bellamy PE, Simmons DH. Diaphragmatic contraction during assisted mechanical ventilation. Chest 1989;96:130-5.

14 Branson RD, Campbell RS, Davis K, Johnson DJ. Comparison of pressure and flow triggering systems during
continuous positive airway pressure. Chest $1994 ; 106: 540-4$.

15 Giuliani R, Mascia L, Recchia F, Caracciolo A, Fiore T, Ranieri VM. Patient-ventilator interaction during syn-
chronized intermittent mandatory ventilation - effects of chronized intermittent mandatory ventilation - effects of

16 Iber C, Simon P, Skatrud JB, Mahowald MW, Dempsey JA. The Breuer-Hering reflex in humans: effects of pulmonary denervation and hypocapnia. Am $\mathcal{F}$ Respir Crit Care Med 1995;152:217-224.

17 Fitting JW. Respiratory muscles during ventilatory support. Eur Respir $\mathcal{F}$ 1994;7:2223-5.

18 McCool FD, Mayewski RF, Shayne DS, Gibson CJ, Griggs RC, Hyde RW. Intermittent positive pressure breathing in patients with respiratory muscle weakness: alterations in patients with respiratory muscle weakness: alterations

19 Pillet O, Choukroun ML, Castaing Y. Effects of inspiratory flow rate alterations on gas exchange during mechanical ventilation in normal lungs: efficiency of end-inspiratory ventilation in normal lungs: effici
pause. Chest 1993;103:1161-5.

20 Leger P, Bedicam JM, Cornette A, Reybet-Degat O, Langevin $\mathrm{B}$, Polu JM, et al. Nasal intermittent positive pressure ventilation: long-term follow-up in patients with severe chronic respiratory insufficiency. Chest 1994;105:100-105.

21 Fabry B, Guttmann J, Eberhard L, Bauer T, Haberthur C, Wolff $G$. An analysis of desynchronization between the spontaneously breathing patient and ventilator during inspiratory pressure support. Chest 1995;107:1387-94.

22 Corbridge TC, Hall JB. The assessment and management of adults with status asthmaticus. Am $\mathcal{f}$ Respir Crit Care Med 1995;151:1296-316.

23 Jubran A, Van De Graaff WB, Tobin MJ. Variability of patient-ventilator interaction with pressure support ventilation in patients with chronic obstructive pulmonary disease. Am $₹$ Respir Crit Care Med 1995;152:129-36. 